\title{
Erratum to: Optical Gain of 1550-nm Range Multiple- Quantum-Well Heterostructures and Limiting Modulation Frequencies of Vertical-Cavity Surface-Emitting Lasers Based on Them
}

E. S. Kolodeznyi ${ }^{a}$, S. S. Rochas ${ }^{a}$, A. S. Kurochkin ${ }^{a}$, A. V. Babichev ${ }^{a}$, I. I. Novikov ${ }^{a}$ *, A. G. Gladyshev ${ }^{a}$, L. Ya. Karachinsky ${ }^{b, c}$, D. V. Denisov ${ }^{b, d}$, Yu. K. Bobretsova ${ }^{c}$, A. A. Klimov ${ }^{c}$, S. A. Blokhin ${ }^{c}$, K. O. Voropaeve, , and A. S. Ionov

${ }^{a}$ ITMO University, St. Petersburg, 197101 Russia

${ }^{b}$ Connector Optics LLC, St. Petersburg, 194292 Russia

${ }^{c}$ Ioffe Physical Technical Institute, Russian Academy of Sciences, St. Petersburg, 194021 Russia

${ }^{d}$ St. Petersburg Electrotechnical University, St. Petersburg, 197376 Russia

${ }^{e}$ Yaroslav-the-Wise Novgorod State University, Veliky Novgorod, 173003 Russia

${ }^{f}$ OKB-Planeta, Veliky Novgorod, 173004 Russia

*e-mail: innokenty.novikov@connector-optics.com

Received September 19, 2018

DOI: $10.1134 / \mathrm{S} 0030400 \mathrm{X} 18100272$

The name of the seventh author should read L. Ya. Karachinsky.

The original article can be found online at https://doi.org/10.1134/S0030400X18080143 